\title{
Late Onset Subacute Profound Biotinidase Deficiency Caused by a Novel Homozygous Variant c.466-3T>G in the BTD Gene
}

\author{
Kaustubh Mohite ${ }^{1} \cdot$ Karthik Vijay Nair $^{2} \cdot$ Anilkumar Sapare $^{1} \cdot$ Venkatraman Bhat $^{3} \cdot$ Anju Shukla $^{2} \cdot$ Minal Kekatpure $^{4}$. \\ Siddaramappa J. Patil ${ }^{5}$ (I)
}

Received: 13 May 2021 / Accepted: 3 September 2021 / Published online: 14 January 2022

(c) Dr. K C Chaudhuri Foundation 2021

\begin{abstract}
Biotinidase deficiency (BD) is an autosomal recessive disorder caused by bi-allelic mutation in the BTD gene. Clinical manifestations in BD mainly depends on residual biotinidase enzyme activity, although there are some exceptions. Broadly BD disorders are classified as profound BD and partial BD. Further profound BD can be early onset, late onset, and sometimes may be asymptomatic. Clinically late-onset profound BD can present with spectrum of manifestations ranging from single organ to multiple organ involvement, typically affecting function of brain, eye, ear, and skin. Here, a first-born child to consanguineous parents with late-onset profound $\mathrm{BD}$ presenting with hyperventilation secondary to lactic acidosis, hypotonia, evolving spasticity, and abnormal neuroimaging findings caused by novel homozygous variant, c.466-3T $>\mathrm{G}$ in the BTD gene is reported.
\end{abstract}

Keywords Biotinidase deficiency $\cdot$ Hyperventilation $\cdot$ Weakened splice variant $\cdot 3$-hydroxyisovalerate $\cdot$ Lactic acidosis

\section{Introduction}

Inherited disorders of biotin metabolism can be due to defective recycling of biotin, holocarboxylase synthetase deficiency, and rarer defective transporter defect of biotin [1,2]. Defective recycling of biotin is due to biotinidase enzyme (BE) deficiency. The clinical manifestations of biotinidase deficiency (BD) depend upon residual enzyme activity.

Siddaramappa J. Patil

drsjpatil@gmail.com

1 Department of Pediatrics, Mazumdar Shaw Medical Center, Narayana Hrudayalaya Hospitals, Bangalore, Karnataka, India

2 Department of Medical Genetics, Kasturba Medical College, Manipal Academy of Higher Education, Manipal, Karnataka, India

3 Department of Radiology, Mazumdar Shaw Medical Center, Narayana Hrudayalaya Hospitals, Bangalore, Karnataka, India

4 Department of Neurology, Division of Pediatric Neurology, Mazumdar Shaw Medical Center, Narayana Hrudayalaya Hospitals, Bangalore, Karnataka, India

5 Division of Medical Genetics, Mazumdar Shaw Medical Center, Narayana Hrudayalaya Hospitals, Bangalore, Karnataka 560099, India
Profound BE (defined as $<10 \%$ of residual BE activity) clinically manifests early in infancy with progressive neurological features, respiratory manifestations, eye involvement, hearing loss, cutaneous findings, and various underlying biochemical findings. Profound symptomatic BD, if untreated, can lead to coma and death $[1,3]$. In some individuals profound $\mathrm{BD}$ clinical presentation can be delayed from late childhood to adolescence and adulthood [1]. Here, subacute late-onset presentation of profound BD caused by novel bi-allelic variant c. $466-3 \mathrm{~T}>\mathrm{G}$ in the $B T D$ is presented.

\section{Case Report}

Apparently healthy 1-y-3-mo old female born to consanguineous parents (Fig. 1A) presented with history of recurrent episodes of cough and cold for the last $3 \mathrm{mo}$. She was referred to us with severe respiratory distress of 1-d duration. Investigations showed no pulmonary pathology, and COVID-19 RT-PCR was negative. ABG showed metabolic acidosis with high lactate $[4.6 \mathrm{mmol} / \mathrm{L}(0.5-1.6)]$.

Her head circumference was $44 \mathrm{~cm}$ (3-5th centile), length was $80.5 \mathrm{~cm}$ (60-70th centile), and weight was $10.6 \mathrm{~kg}$ (60th centile). CNS examination showed hypotonia with presence of deep tendon reflexes and eye evaluation showed increased slow saccades. 


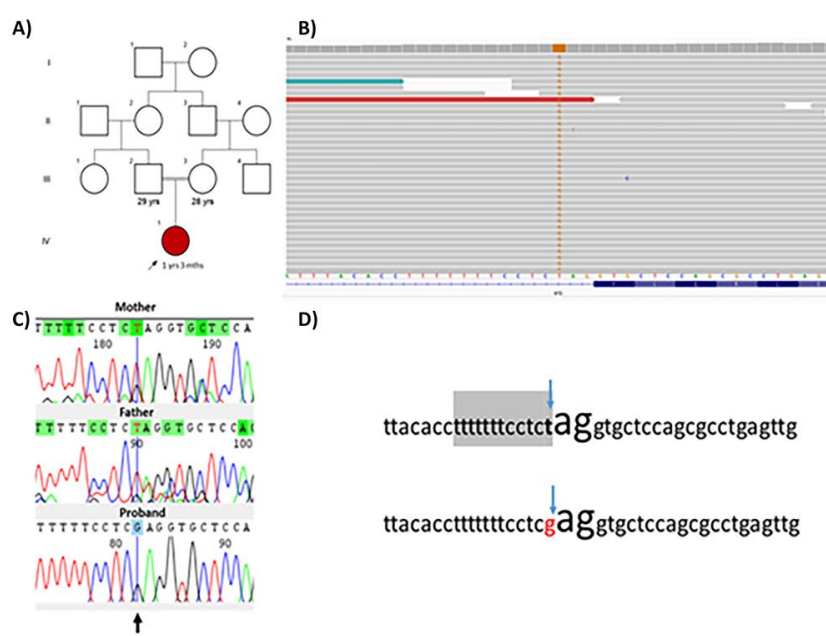

Fig. 1 A) Four generation pedigree showing inheritance pattern. B) Visualization of novel $B T D$ variant in Integrative Genomic Viewer. C) Familial segregation analysis showing (black arrow) the variant c.466-3T $>\mathrm{G}$ in homozygous state in affected child and in heterozygous state in parents. D) Intron 3 canonical acceptor splice site (in higher font) along with its proximal consensus sequences (grey highlight in top sequences). Top sequences showing normal sequences and bottom sequences showing the variant (highlighted in red) $(-3 \mathrm{~T}>\mathrm{G})$. (a) $\mathrm{T} 2 \mathrm{~W}$ axial and (b) FLAIR images demonstrating hyperintense areas in the under surface of rostrum of corpus callo-

Her CBC, liver function, and thyroid functions tests were normal. Her LC-MSMS blood acylcarnitine profile showed elevation of C4-DC $[2.57 \mu \mathrm{mol} / \mathrm{L}$ (normal range $<1.29 \mu \mathrm{mol} / \mathrm{L}$ ) $]$ and $\mathrm{C} 5-\mathrm{OH}[1.54 \mu \mathrm{mol} / \mathrm{L}$ (normal range $<0.9)]$. Urine organic acid analyses showed elevation of lactic acid along with elevation of other metabolites of Krebs cycle, amino acids, and acylglycines.

MR of brain showed T2 and FLAIR hyperintense signals (HS) with diffusion restriction in diffusion weighted images (DWI) along the under surface of the rostrum of the corpus callosum, adjacent septum pellucidum, and fornix (Fig. 1a-c), T2 weighted axial image demonstrated normal signals of cerebellum and brainstem (Fig. 1d), and the columns of fornix to hippocampal commissure (Fig. 1e-g). Additional HS were noted in the periaqueductal area, the dorsal region of upper cervical cord (Fig. 1c), and focal area of diffusion restriction at the grey-white matter junction of left cerebellum (Fig. 1g).

After discharge, her achieved motor milestones were lost - unable to sit and have a steady neck control, along with evolving spasticity in the limbs.

BE assay showed low BE levels of $0.8 \mathrm{nmol} / \mathrm{min} / \mathrm{mL}$ (8.8\%) [4.1-14.5 (mean \pm SD 9.09 \pm 4.48$)]$. Whole exome sequencing (WES) identified a rare intronic variant, c.466-3T $>\mathrm{G}$ (NC_000003.11:g.15685820T $>$ G) in the BTD gene (GenBank: NM_001281724) in homozygous (Fig. 1B). Sanger analysis confirmed the autosomal recessive segregation (Fig. 1C).

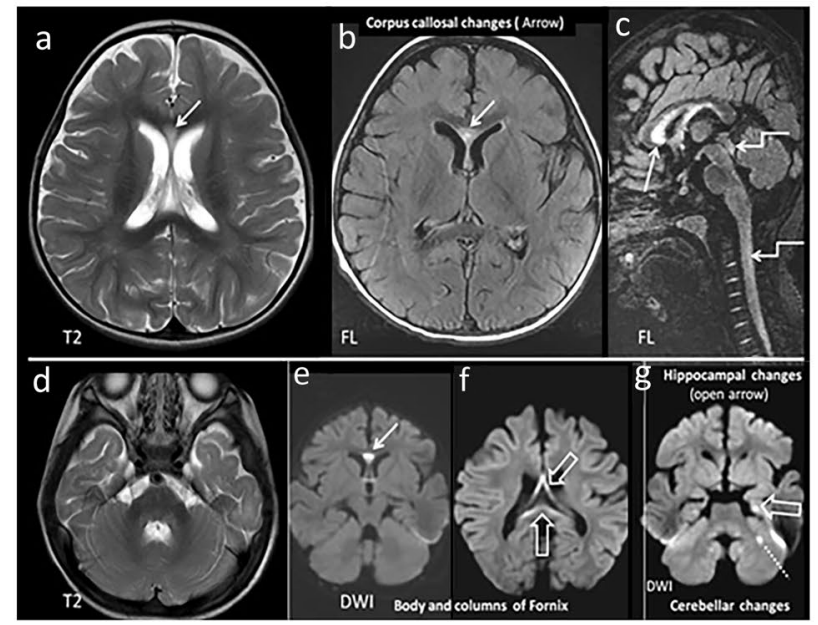

sum (arrows) and adjacent septum pellucidum and fornix. (c) Sagittal FLAIR image showing the complete extent of the changes (arrow) and some additional changes in periaqueductal area and dorsal region of upper cervical cord (elbow arrow). (d) T2 weighted axial image demonstrated normal signals of cerebellum and brainstem. (e-g) Involved regions showing diffusion restriction-restricted diffusion extends along columns of fornix to hippocampal commissars (open arrows). Focal area of diffusion restriction is also noted at grey-white matter junction of cerebellum (dotted line)

\section{Discussion}

$\mathrm{BD}$ is as autosomal recessive disorder with an estimated combined (profound and partial) incidence of 1 in 61,000 [1]. In some population studied with the use of newborn screening, incidence of BD has been found to be higher than the above figures [4-6].

The clinical manifestations of late-onset untreated profound $\mathrm{BD}$ (defined here as $>1 \mathrm{y}$ age) range from seizures, hypotonia, progressive spastic paraparesis, ataxia, developmental delay, cutaneous manifestations, vision loss (optic atrophy), sensorineural hearing loss, respiratory symptoms, recurrent infections (viral and fungal) to asymptomatic adults $[1,3]$. Variable age presentation of profound BD has been attributed to various genetic and nongenetic factors. The present patient presented with respiratory symptoms along with hypotonia. Respiratory manifestations seen in BD include hyperventilation, laryngeal stridor, apnea, and recurrent respiratory infections [1].

In profound $\mathrm{BD}$, the findings of the brain imaging include cerebral or cerebellar atrophy, cerebral edema, delayed myelination, dilated ventricles, and ischemic areas seen as HS/diffusion restriction in DWI in the various parts of the brain. In addition, some patients can show HS changes in T2 and FLAIR images in the spinal cord suggestive of myelopathy $[3,6-8]$. The present patient showed 
both brain and spinal cord abnormal imaging findings seen in $\mathrm{BD}$.

BE replenishes biotin pool by recycling biotin. BD leading to biochemical abnormalities include lactic acidosis, hyperammonemia, and various organic acid metabolites due to multiple carboxylases deficiencies. Increased lactate and increased 3-hydroxyisovalerate were seen in the present patient with other metabolites of carboxylases deficiencies.

WES identified a rare splice homozygous pathogenic variant, c.466-3T $>\mathrm{G}$, and was present in the sequences surrounding the canonical acceptor splice site of intron 3 (Fig. 1D). Splicing defects in the consensus sequence surrounding the canonical splice sites reduce strength of natural splice sites (weakened splice sites). Weakened splice sites can exhibit normal mRNA as well as lower levels of altered mRNA [9].

The patient was started on $10 \mathrm{mg}$ of biotin daily. There was dramatic improvement of her clinical abnormalities. Although there are many cases of $\mathrm{BD}$ from India, there are very few reports of molecular studies [10]. Variable clinical presentation makes BD diagnosis challenging and rewarding, especially in countries where newborn screening is not universal.

\section{Conclusion}

In conclusion, this patient with profound BD illustrates a respiratory mimic caused by the novel $B T D$ variant.

Note: BE enzyme levels should be used for diagnosis of typical BD cases along with single gene sequencing. WES was utilized as an initial test, in view of atypical presentation for BD.

Acknowledgements The authors are thankful to the family for participation in the study.

Authors' Contributions $\mathrm{KM}$ and AkS drafted the initial manuscript; KVN and AS contributed towards molecular methods and analyses;
VB: Radiology-related images and discussion; MK: Neurological management and discussion; SJP drafted the final manuscript.

Guarantor Dr Rajiv Aggarwal, Head, Department of Pediatrics, Mazumdar Shaw Medical Center, Narayana Hrudayalaya Hospitals, Bangalore.

\section{Declarations}

Conflict of Interest None.

\section{References}

1. Wolf B. Biotinidase deficiency: "if you have to have an inherited metabolic disease, this is the one to have." Genet Med. 2012;14:565-75.

2. Mardach R, Zempleni J, Wolf B, et al. Biotin dependency due to a defect in biotin transport. J Clin Invest. 2002;109:1617-23.

3. Wolf B. The neurology of biotinidase deficiency. Mol Genet Metab. 2011;104:27-34.

4. Jay AM, Conway RL, Feldman GL, Nahhas F, Spencer L, Wolf B. Outcomes of individuals with profound and partial biotinidase deficiency ascertained by newborn screening in Michigan over 25 years. Genet Med. 2015;17:205-9.

5. Funghini S, Tonin R, Malvagia S, et al. High frequency of biotinidase deficiency in Italian population identified by newborn screening. Mol Genet Metab Rep. 2020;25:100689.

6. Canda E, Kalkan Uçar S, Çoker M. Biotinidase deficiency: prevalence, impact and management strategies. Pediatric Health Med Ther. 2020;11:127-33.

7. Bhat MD, Bindu PS, Christopher R, Prasad C, Verma A. Novel imaging findings in two cases of biotinidase deficiency-a treatable metabolic disorder. Metab Brain Dis. 2015;30:1291-4.

8. Shah S, Khan N, Lakshmanan R, Lewis B, Nagarajan L. Biotinidase deficiency presenting as neuromyelitis optica spectrum disorder. Brain Dev. 2020;42:762-6.

9. Mucaki EJ, Shirley BC, Rogan PK. Expression changes confirm genomic variants predicted to result in allele-specific, alternative mRNA splicing. Front Genet. 2020;11:109.

10. Raha S, Udani V. Biotinidase deficiency presenting as recurrent myelopathy in a 7-year-old boy and a review of the literature. Pediatr Neurol. 2011;45:261-4.

Publisher's Note Springer Nature remains neutral with regard to jurisdictional claims in published maps and institutional affiliations. 